\title{
BMJ Open Standardising neonatal and paediatric antibiotic clinical trial design and conduct: the PENTA-ID network view
}

\author{
Laura Folgori (D) ,1,2 Irja Lutsar, ${ }^{3}$ Joseph F Standing, ${ }^{1,4}$ A Sarah Walker, ${ }^{5,6}$ \\ Emmanuel Roilides, ${ }^{7}$ Theoklis E Zaoutis, ${ }^{8}$ Hasan Jafri, ${ }^{9}$ Carlo Giaquinto, ${ }^{10}$ \\ Mark A Turner, ${ }^{11}$ Mike Sharland ${ }^{1}$
}

To cite: Folgori L, Lutsar I, Standing JF, et al. Standardising neonatal and paediatric antibiotic clinical trial design and conduct: the PENTA-ID network view. BMJ Open 2019;9:e032592. doi:10.1136/ bmjopen-2019-032592

- Prepublication history for this paper is available online. To view these files, please visit the journal online (http://dx.doi. org/10.1136/bmjopen-2019032592).

Received 26 June 2019 Revised 28 November 2019 Accepted 29 November 2019

Check for updates

(c) Author(s) (or their employer(s)) 2019. Re-use permitted under CC BY-NC. No commercial re-use. See rights and permissions. Published by BMJ.

For numbered affiliations see end of article.

Correspondence to Dr Laura Folgori; Ifolgori@sgul.ac.uk

\section{ABSTRACT}

Antimicrobial development for children remains challenging due to multiple barriers to conducting randomised clinical trials (CTs). There is currently considerable heterogeneity in the design and conduct of paediatric antibiotic studies, hampering comparison and meta-analytic approaches. The board of the European networks for paediatric research at the European Medicines Agency (EMA), in collaboration with the Paediatric European Network for Treatments of AIDSInfectious Diseases network (www.penta-id.org), recently developed a Working Group on paediatric antibiotic CT design, involving academic, regulatory and industry representatives. The evidence base for any specific criteria for the design and conduct of efficacy and safety antibiotic trials for children is very limited and will evolve over time as further studies are conducted. The suggestions being put forward here are based on the adult EMA guidance, adapted for neonates and children. In particular, this document provides suggested guidance on the general principles of harmonisation between regulatory and strategic trials, including (1) standardised key inclusion/ exclusion criteria and widely applicable outcome measures for specific clinical infectious syndromes (CIS) to be used in CTs on efficacy of antibiotic in children; (2) key components of safety that should be reported in paediatric antibiotic CTs; (3) standardised sample sizes for safety studies. Summarising views from a range of key stakeholders, specific criteria for the design and conduct of efficacy and safety antibiotic trials in specific CIS for children have been suggested. The recommended criteria are intended to be applicable to both regulatory and clinical investigator-led strategic trials and could be the basis for harmonisation in the design and conduct of CTs on antibiotics in children. The next step is further discussion internationally with investigators, paediatric CTs networks and regulators.

\section{WHAT IS THE PROBLEM?}

Antimicrobial resistance (AMR) is a rapidly emerging problem, causing morbidity and mortality especially in vulnerable populations. Mortality attributable to AMR may be associated with discordant therapy, which is particularly challenging in neonates and children due to the limited number of approved effective antimicrobials, the inadequate pipeline for novel antibiotics and the long delays noted in many documents between the adult and paediatric licensing of novel antibiotics. $^{12}$ There is no evidence that the significant delays in paediatric licensing of new antibiotics is improving. Antimicrobial development for children remains challenging due to multiple barriers to conducting clinical trials (CTs), with nearly half of paediatric medicines in Europe prescribed off-label, without evidence on the optimal dosage or safety data. ${ }^{3}$ The Clinical Trials Transformation Initiative, aiming to develop and drive adoption of practices that will increase the quality and efficiency of CTs, recently organised a Multi-Stakeholder Expert Meeting with the aim to identify and address barriers in conducting antibacterial CTs in neonates and children. ${ }^{2}$ We have previously reported on the marked heterogeneity in the design and conduct of paediatric antibiotic trials, with a lack of standardisation of the key inclusion/exclusion criteria and endpoints for specific clinical infectious syndromes (CIS) hampering comparison between studies and meta-analytic approaches. ${ }^{4}$

Among the initiatives put in place to improve the efficiency and feasibility of paediatric CTs was the publication of a Paediatric Addendum to the Guideline on the evaluation of medicinal products indicated for treatment of bacterial infections ${ }^{5}$ by the Committee for Medicinal Products for Human Use and the Infectious Diseases Working Party at the European Medicines Agency (EMA). The purpose of this publication was to provide some general consideration on the paediatric clinical development programmes required to support the authorisation of antibacterials for treatment of infectious diseases in children AND in the optimal design and conduct of clinical investigator-led strategic trials. ${ }^{6}$ The board of the 
European networks for paediatric research at the EMA, in collaboration with the Paediatric European Network for Treatments of AIDS-Infectious Diseases network, therefore developed a Working Group (WG) on paediatric antibiotic CT design, involving academic, regulatory and industry representatives from both the USA and Europe. This group aimed to provide practical guidance on the design and conduct of neonatal and paediatric antibiotic CTs in order to improve international harmonisation in this important area. Currently, the EMA recommends the conduct of single-dose/multi-dose pharmacokinetic (PK) studies to support the approval of an antibacterial agent to treat infectious diseases in paediatric patients. ${ }^{5}$

The evidence base for any specific criteria for the design and conduct of efficacy and safety antibiotic trials for children is very limited and will evolve over time as further studies are conducted. The suggestions being put forward here are based on the adult EMA guidance, adapted for neonates and children. ${ }^{6-8}$ In particular, this document provides suggested guidance on the general principles of harmonisation between regulatory and strategic trials, including:

1. Standardised key inclusion/exclusion criteria and widely applicable outcome measures for specific CIS to be used in CTs on efficacy of antibiotic in children.

2. Key components of safety that should be reported in paediatric antibiotic CTs.

3. Standardised sample sizes for safety studies.

\section{GENERAL PRINCIPLES}

There are clear differences between regulatory trials being conducted to obtain a marketing authorisation for a new molecular entity and strategic trials usually sponsored by academic institutions. However, where possible, similar standards should apply across both types of studies. This distinction is becoming less wide as collaboration between clinical academic CT networks and pharma to drive efficiency increases, with both groups committed to the more rapid delivery of high quality trials. ${ }^{2}$ Recent data has noted that there has been in general inadequate reporting of safety in investigator-led paediatric antibiotic CTs and marked heterogeneity of the key trial elements, for example, inclusion/exclusion criteria and definition and timing of end points. ${ }^{34}$

There is increasing recognition that for the great majority of paediatric regulatory antibiotic trials, for well-established classes, both efficacy and safety can be bridged from adult studies. Single-dose PK studies are difficult to perform and there needs to be a clear focus on reducing barriers to recruitment. In our view, singledose PK studies do not need to be conducted only in the CIS where there is an adult licence but will recruit more efficiently as an 'all comers' study where the child may be in hospital with any CIS. We can see no scientific rationale why the PK for the great majority of antibiotics (eg, a beta lactam/beta lactam inhibitor combination-BL/ BLI) will be different if the child is stable and completing a course of antibiotics for complicated urinary tract infection, or a complicated intra-abdominal infection. The child should be clinically stable, on either intravenous or oral antibiotics, being given for treatment or prophylaxis. If the antibiotic has very predictable linear PK and a well described safety profile, then the cohorts across all ages, including neonates, should be opened at the same time. Wherever possible, the neonatal single-dose PK cohort should be included in the same protocol as the older age cohorts, as separate protocols may lead to significant delays in determining the neonatal dose.

The scientific rationale for a multi-dose study needs to be determined on a case by case basis. As can be seen from the sample sizes given below, multi-dose studies of less than around 100 children for well-established classes of antibiotics will not be adequately powered to determine any new safety signal that is not entirely predictable from that drug class. Some antibiotics that, for example, require a loading dose calculated from the single-dose PK study, will need a multi-dose validation PK study. There may be a rationale for certain novel antibiotics to gain experience of routine clinical use from an open label, all comers, multi-dose treatment study, while recognising that the study is not required for PK and is not powered for either safety or efficacy. There does not appear to be any clear scientific rationale for the great majority of well-established classes of antibiotics for randomisation between the novel agent and a standard of care (SOC) $\mathrm{arm}$. Even if SOC can be controlled to a limited number of regimens (which is often difficult in studies requiring multiple sites to achieve the recruitment targets), as seen from the sample sizes given below, the trial would need to be recruiting a very substantial number of children to detect any novel safety signal that was not entirely predictable from the drug class. It should be emphasised that these comments only apply to well-established drug classes (eg, BL/BLIs, aminoglycosides, etc).

The optimal study design for neonates is evolving and requires further international consensus. There is an urgent global unmet clinical need for novel antibacterial agents to treat neonates, both term and preterm, with multidrug resistant bacterial infection causing neonatal sepsis. Equally, not every new antibiotic under development needs evaluation in neonates, where, for example, there are already other treatment options. For many new antibiotics single-dose PK studies would be all that would reasonably be required.

The next step would be a prioritisation of the novel antibiotics that are a high priority for multi-dose safety and efficacy trials in neonates. This could be based on the WHO Priority Pathogen List, focussing on the most critical pathogens, specifically those agents active against carbapenem resistant organisms. ${ }^{9}$ For these relatively few antibiotics, PK, safety and efficacy data are required in the indication of neonatal serious/severe bacterial infection (SBI, sepsis). Evaluation of penetration of the drug into the central nervous system for these antibiotics is also required. With the majority of neonatal sepsis caused by 
multidrug resistant Gram-negative pathogens, much of which is healthcare-associated, there is no obvious scientific basis to divide neonatal sepsis into early and late onset sepsis and the general term neonatal serious bacterial infection is the most suitable term (as used by the WHO). These studies will need to recruit babies across all stages of prematurity and postnatal age. Given the challenges of recruitment into such a population and the need for such trials to recruit globally, active consideration should be given for establishing close collaboration between Pharma, the WHO, global paediatric infectious diseases CTs networks and other major stakeholders, similar to the structures that were developed for paediatric HIV infection. ${ }^{10}$ Novel trial designs need to be urgently considered allowing the inclusion of multiple agents within protocols, focussing on obtaining both regulatory and public health outcomes within single trials. We urgently recommend the WHO to convene a consensus meeting focused specifically on neonatal sepsis to drive forward the global collaboration required.

The reporting of pharmacovigilance data on antibiotics in neonates and children is currently limited. At the moment, a standardised method of conducting antibiotic pharmacovigilance in children and neonates has not been developed, particularly for drugs that are used offlabel. This is an increasingly important area for all medicines as key regulatory trials have smaller sample sizes related to cost considerations. The establishment of a network of different stakeholders (academics, physicians, regulators and industry) involving centres in all regions across the world would allow the conduct of prospective cohort studies using electronic data records as part of post-marketing surveillance (as has already been set up with paediatric HIV registries). Such approach could potentially allow data to be collected and easily pooled out at a relatively low cost. ${ }^{11}$

\section{Suggested key clinical and laboratory components of inclusion/exclusion criteria and endpoints for CIS in paediatric antibiotic CTs}

In the absence of any clearly accepted criteria, while recognising the very limited evidence base but given the wide variation seen in reported CTs, the WG has developed suggestions for paediatric inclusion/exclusion criteria and endpoints for the most common CIS.

Based on the results of a recently conducted systematic review, ${ }^{4}$ the most frequently reported CIS-specific clinical and laboratory criteria for the enrolment and evaluation of children in antibiotic CTs were collected. These criteria were then compared with the EMA Guideline on the evaluation of medicinal products indicated for treatment of bacterial infections, ${ }^{6-8}$ revised according to the expert opinion of the WG members, and summarised in table 1. The WG decided to adapt the adult EMA criteria for children and neonates in all those CIS in which a similar pathophysiology and a similar spectrum of pathogens across the target age groups could be anticipated. This has been also the principle that has be adopted in the Paediatric
Addendum for the extrapolation of efficacy against an infectious disease from adults to paediatric patients. ${ }^{5}$ This situation applies to the majority of infectious diseases that occur both in adults and in one or more paediatric age subgroups. However, there are some cases in which the pathophysiology and the spectrum of pathogens differ substantially between children/neonates and adults. As discussed above, for example, this is the case of neonatal sepsis (neonatal SBI). In this case, age-specific criteria have been adopted specifically designed for the neonatal age.

\section{Key components of safety in paediatric antibiotic CTs}

Proper reporting of safety data when publishing clinical studies would increase translation of results into clinical practice. $^{12}$ We have previously published a systematic review of safety data reported in CTs of antibacterial agents in children and neonates to determine if age-specific adverse events (AEs) could be identified for different antibiotic classes. ${ }^{3}$ The quality of reporting AEs was suboptimal in the great majority of CTs, due to the lack of detailed definitions of expected/unexpected AEs (with respect to the AEs that have been reported in adults and/or the mechanism of action of the study drug), grading, reference for Coding System, and age stratification of the results. To improve the quality of safety reporting we recommend that there should be a specific section on safety in every paediatric antibiotic CT. To allow an appropriate comparison between CTs, studies should provide:

- Justification of the sample size for safety, and definition of the safety population in studies having safety as a primary endpoint.

- Definition for:

- How harms-related information was collected (mode of data collection, timing, attribution methods, harms-related monitoring and stopping rules). ${ }^{13}$

- Predefinition of each specific clinical/laboratory/ imaging addressed AE.

- Grading (mild, moderate, severe).

- Relationship with the study drug (expected vs unexpected).

- Reference for Coding System (taking into account that most groups are now using the Division of AIDS grading system). ${ }^{14}$

- Overall (all age groups together) analysis presented first, followed by stratification of safety assessments and results by different age groups.

- Data on any modification to randomised treatment OR withdrawals because of AEs.

- All the denominators and all absolute risks per arms and per AE type, grade, seriousness and severity.

\section{Standardising sample sizes for paediatric antibiotic CTs}

Data obtained from underpowered studies limits the implementation of the result itself, wastes resources, and undermines the ethics of patients' involvement. However, 


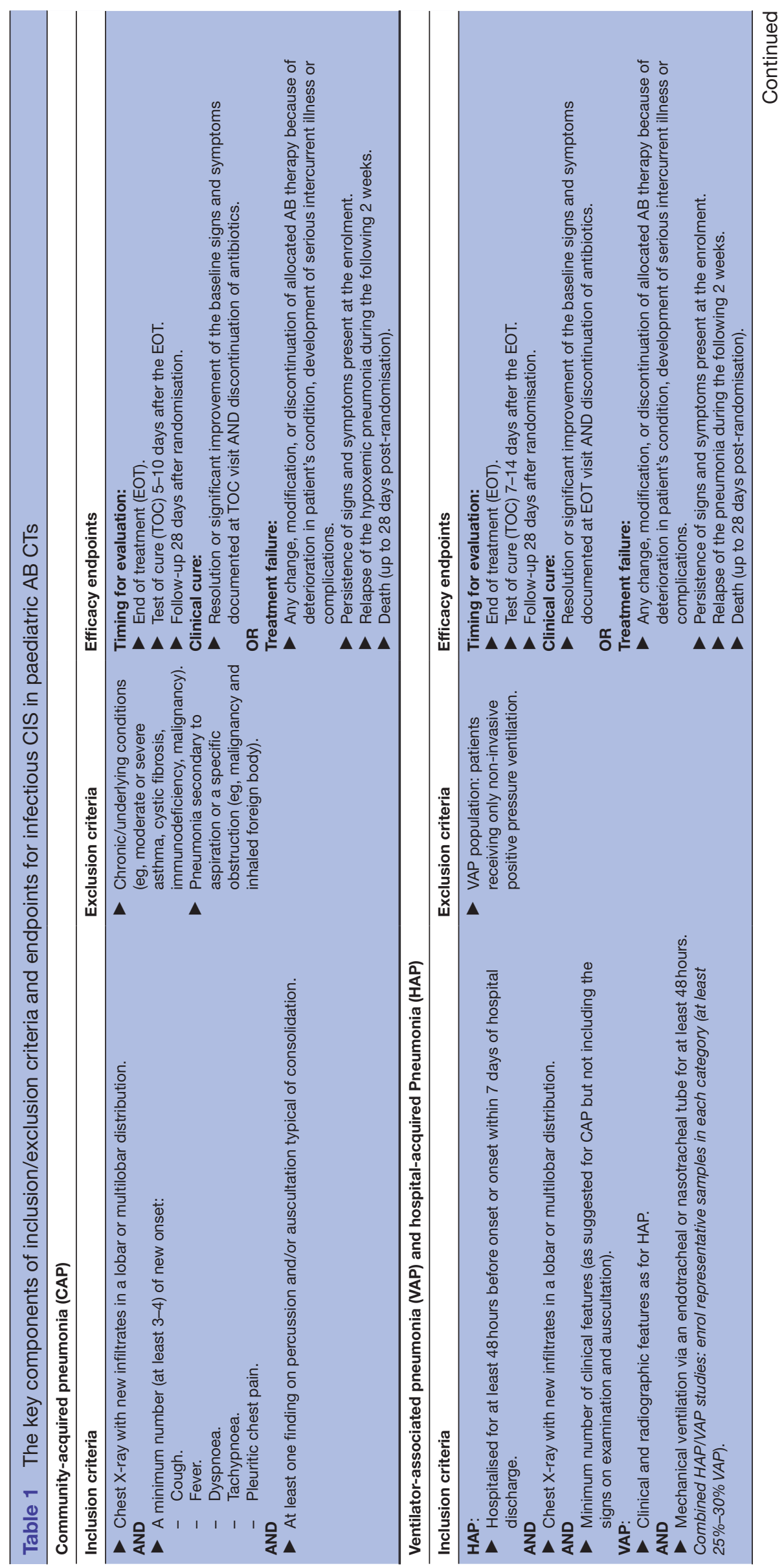




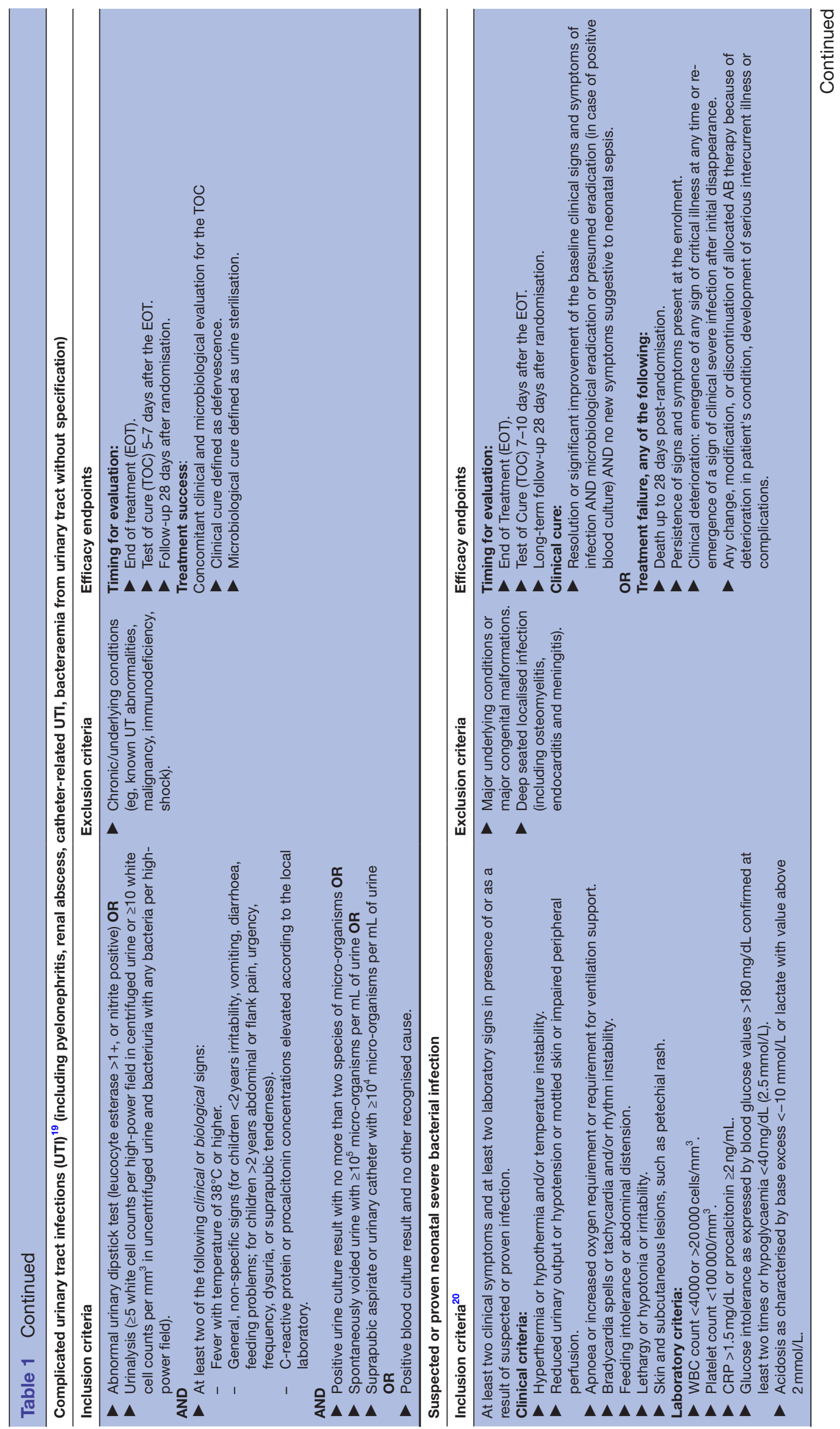




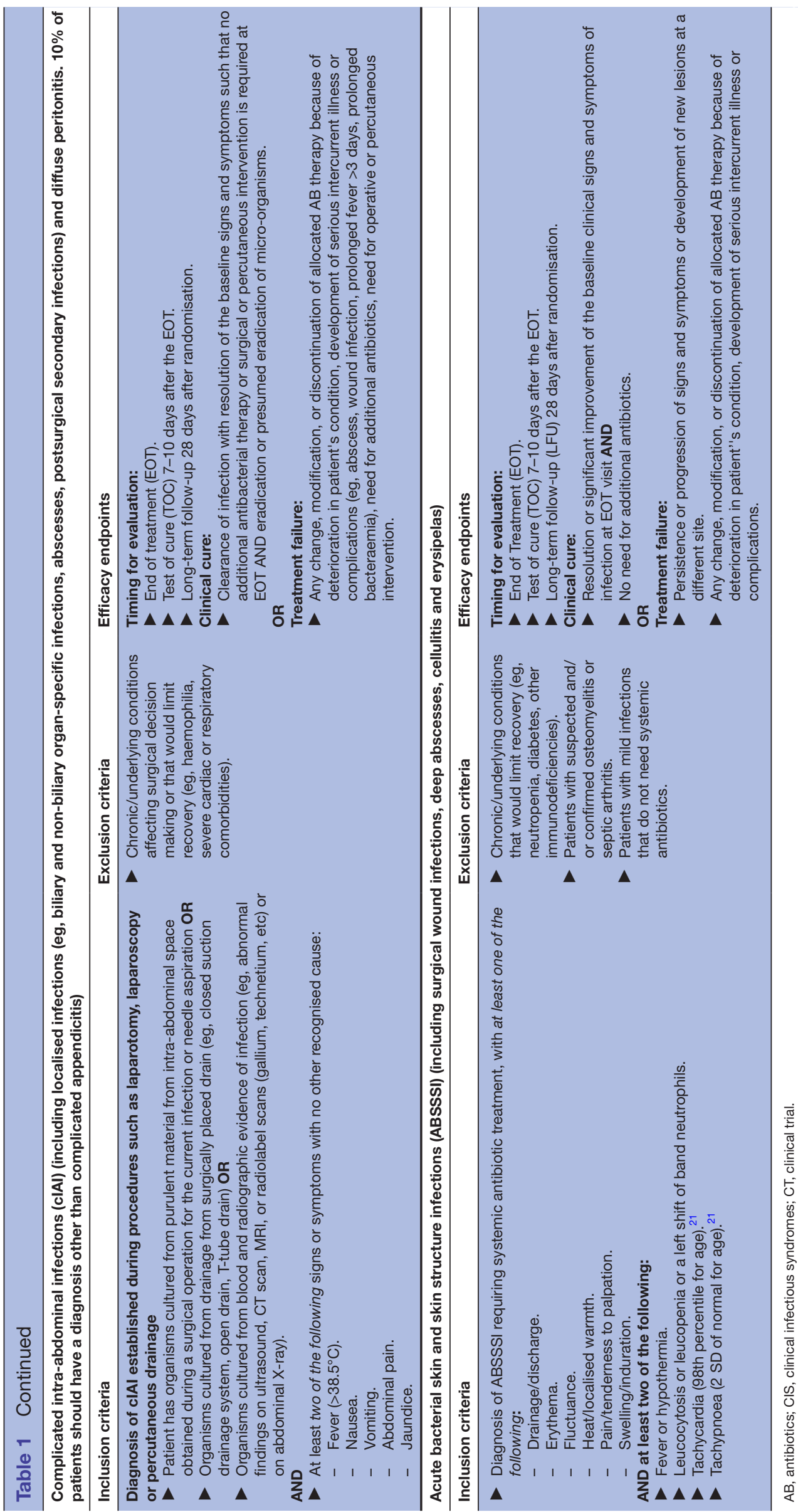

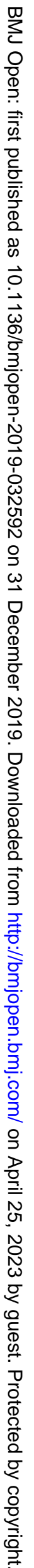







in the safety review, only two trials provided the justification for the sample size specifically for the safety population, including those designed with safety as the primary endpoint.

In an attempt to provide a standardised sample size to be used in single-arm interventional paediatric antibiotic CTs having safety as a primary endpoint, based on the rates of AEs per single drug class reported in the systematic review, the WG considered some key underpinning concepts. First, the rates of AEs and serious AEs (SAEs) in children are generally low, often lower than in adults, and usually predictable by class; AEs/SAEs specific to children occur extremely rarely, but are important to detect; and blinded (placebo-controlled) or unblinded comparative trials aim to estimate the difference between $\mathrm{AE}$ rates with the new antibiotic versus a comparator, with sample sizes typically large if designed to exclude differences outside a non-inferiority margin, or powered only to detect very large reductions in AEs which may not be realistic.

Given this, a reasonable approach would be to ensure sufficient children receive a novel antibiotic to enable (1) a high probability of determining that the overall $\mathrm{AE} / \mathrm{SAE}$ rate is estimated reasonably precisely and (2) a reasonable probability of observing an $\mathrm{AE}$ which occurs in $1 / 20$ children, or equivalently, that an observation of zero events has an upper $97.5 \%$ CI which lies below $5 \%$. This could be done within a single-arm interventional trial with a standard proportion test (as, eg, in Flahault et $a l^{15}$ ). Given an expected proportion of children experiencing one or more AEs, and a maximum acceptable value for this proportion, the sample sizes in table 2 provide the $0.95,0.90$ and 0.80 probability that the upper 95\% CI around the proportion of children experiencing one or more AEs in the new trial is below the maximum acceptable value. An observation of no AEs of a particular kind out of $\mathrm{N}$ children has an upper $97.5 \%$ CI limit which is approximately $3 / \mathrm{N}$ (as a proportion). ${ }^{16}$ For example, for 0 events observed from 60 children, the approximation is $3 / \mathrm{n}=3 / 60=1 / 20=0.05$ (compared with the actual exact upper limit, which is 0.06 ).

A further analysis then considered the potential classspecific sample sizes using data from the safety systematic review discussed above. ${ }^{3}$ In table 2 , the third, fifth and seventh columns represent the sample size that would provide a $>0.80,>0.90$ and $>0.95$ probability, respectively, that the final $95 \% \mathrm{CI}$ around the estimated percentage experiencing AEs in the new trial was no more than $10 \%$ higher than the average rate provided in the second column. The fourth, sixth and eighth columns provide the upper $97.5 \%$ confidence limit around an observation of zero AEs of a particular type from this number of children (ie, the degree of certainty that an $\mathrm{AE}$ that was not observed in the trial genuinely had a low frequency).

These sample sizes are intended to inform investigators of the number of children to be enrolled to adequately power single-arm studies on these antibiotic classes having safety as a primary endpoint.

\section{WHAT NEXT?}

The WG has discussed general principles for the design of studies and put forward practical suggestions on clinical inclusion/exclusion criteria for children for antibiotic trials, where none previously existed. We have also put forward suggestions on how to improve safety reporting.

The collaboration between clinical academic CT networks and pharma is improving. However, one of the barriers in conducting CTs in children is the complexity of the inclusion/exclusion criteria which can be a barrier to recruitment. The group therefore attempted to draft criteria for each CIS that would be as simple and inclusive as possible, to try and encourage as wide an adoption by both clinicians and industry, relying on the fact that investigators are keen to use widely recognised criteria when available. In this process, the regulatory agency will have the responsibility for the approval of CTs designed for obtaining a marketing authorisation for a new molecular entity; on the other side, the sponsor will have the responsibility to ensure that the protocol is designed to be as efficient as possible and reflects the relevant current guidance documents for that specific clinical infection.

Considering the limited data currently available on paediatric pharmacology, it is clear that robust evidence of efficacy and safety of different drugs in children can only be gained if CTs are properly conducted and reported. This issue was raised by Saint-Raymond $e t \mathrm{al}^{17}$ who suggested additional reporting requirements to the 2010 Consolidated Standards of Reporting Trials Statement specifically for trials in children. Data on neonates are even more limited. To improve the quality of reporting and strengthen research in this age group, an extension of the Strengthening the Reporting of Observational Studies in Epidemiology (STROBE) statement for neonatal infection research has been published recently-STROBE-Neonatal Infection. ${ }^{18}$ This would help the process of harmonisation in data collection and reporting, therefore increasing translation of results into clinical practice.

In summary, this document is intended to be complimentary to the draft EMA 'Addendum to the guideline on the evaluation of medicinal products indicated for treatment of bacterial infections to address paediatric-specific clinical data requirements'. The WG focused on those aspects not specifically addressed in the draft Addendum, gathering evidence from both published literature and experience from the networks and members involved. The next step is further discussion internationally with investigators, paediatric CTs networks and regulators, and to work towards a wider harmonisation of trial design and conduct.

\section{Author affiliations}

${ }^{1}$ Paediatric Infectious Diseases Research Group, Institute for Infection and Immunity, St George's University of London, London, UK

${ }^{2}$ Paediatric Infectious Disease Unit, Department of Paediatrics, Luigi Sacco Hospital, University of Milan, Milan, Italy

${ }^{3}$ Department of Microbiology, Faculty of Medicine, University of Tartu, Tartu, Estonia 
${ }^{4}$ Great Ormond Street Institute of Child Health, University College London, London, UK

${ }^{5}$ Nuffield Department of Clinical Medicine; NIHR Oxford Biomedical Research Centre, University of Oxford, Oxford, UK

${ }^{6}$ MRC Clinical Trials Unit at UCL, Institute of Clinical Trials Methodology, UCL, London, UK

${ }^{7}$ Infectious Diseases Unit, 3rd Department of Paediatrics, Faculty of Medicine, Aristotle University 96 School of Health Sciences, Thessaloniki, Greece

${ }^{8}$ Division of Infectious Diseases and the Center for Pediatric Clinical Effectiveness, Children's Hospital of Philadelphia, Philadelphia, Pennsylvania, USA

${ }^{9}$ AstraZeneca, 950 Wind River Ln, Gaithersburg, MD, USA

${ }^{10}$ Department of Woman's and Child's Health, University of Padova, Padua, Italy

${ }^{11}$ Institute of Translational Medicine, Centre for Women's Health Research, Liverpool Women's Hospital, Crown Street, Liverpool, UK

Contributors LF and MS contributed to the concept and design of the work. LF, IL, JFS, SW, ER, TEZ, HJ, CG, MAT and MS, as part of the Working Group, contributed to the drafting of the suggested criteria. LF, MS, SW and IL wrote the first draft of the manuscript. All authors reviewed and contributed to subsequent drafts and approved the final version for publication.

Funding The authors have not declared a specific grant for this research from any funding agency in the public, commercial or not-for-profit sectors.

Competing interests None declared.

Patient consent for publication Not required.

Provenance and peer review Not commissioned; externally peer reviewed.

Open access This is an open access article distributed in accordance with the Creative Commons Attribution Non Commercial (CC BY-NC 4.0) license, which permits others to distribute, remix, adapt, build upon this work non-commercially, and license their derivative works on different terms, provided the original work is properly cited, appropriate credit is given, any changes made indicated, and the use is non-commercial. See: http://creativecommons.org/licenses/by-nc/4.0/.

ORCID iD

Laura Folgori http://orcid.org/0000-0002-0680-0450

\section{REFERENCES}

1 Folgori L, Bielicki J, Heath PT, et al. Antimicrobial-resistant gramnegative infections in neonates: burden of disease and challenges in treatment. Curr Opin Infect Dis 2017;30:281-8.

2 Noel GJ, Nambiar S, Bradley J, et al. Advancing pediatric antibacterial drug development: a critical need to Reinvent our approach. J Pediatric Infect Dis Soc 2019;8:60-2.

3 Pansa P, Hsia Y, Bielicki J, et al. Evaluating safety reporting in paediatric antibiotic trials, 2000-2016: a systematic review and metaanalysis. Drugs 2018;78:231-44.

4 Folgori L, Bielicki J, Ruiz B, et al. Harmonisation in study design and outcomes in paediatric antibiotic clinical trials: a systematic review. Lancet Infect Dis 2016;16:e178-89.

5 European Medicines Agency. Addendum to the guideline on the evaluation of medicinal products indicated for treatment of bacterial infections to address paediatric-specific clinical data requirements (EMA/CHMP/187859/2017). Draft. 2018, 2018. Available: https:// www.ema.europa.eu/documents/scientific-guideline/draftaddendum-guideline-evaluation-medicinal-products-indicatedtreatment-bacterial-infections_en.pdf [Accessed 14 Aug 2019].

6 Eurepean Medicines Agency. Guideline on the evaluation of medicinal products indicated for treatment of bacterial infections (CPMP/EWP/558/95 rev 2). Available: http://www.ema.europa. eu/docs/en GB/document library/Scientific guideline/2009/09/ WC500003417.pdf [Accessed 14 Aug 2019].

7 European Medicines Agency. Addendum to the guideline on the evaluation of medicinal products indicated for treatment of bacterial infections (EMA/CHMP/351889/2013). Available: http://www.ema. europa.eu/docs/en_GB/document_library/Scientific_guideline/2013/ 11/WC500153953.pdf [Accessed 14 Aug 2019].

8 European Medicines Agency. Guideline on the evaluation of medicinal products indicated for treatment of bacterial infections Rev. 3 (EMA/844951/2018). Draft, 2018. Available: https://www.ema. europa.eu/documents/scientific-guideline/draft-guideline-evaluationmedicinal-products-indicated-treatment-bacterial-infections-revision3_en.pdf [Accessed 14 Aug 2019].

9 World Health Organization. Global priority list of antibiotic-resistant bacteria to guide research, discovery, and development of new antibiotics, 2017. Available: https://www.who.int/medicines/ publications/WHO-PPL-Short_Summary_25Feb-ET_NM_WHO.pdf? $\mathrm{ua}=1$ [Accessed 14 Aug 2019].

10 Penta child health research. Available: https://penta-id.org/ [Accessed 14 Aug 2019].

11 Sharland M, Rodvold KA, Tucker HR, et al. Safety and efficacy of tigecycline to treat multidrug-resistant infections in pediatrics: an evidence synthesis. Pediatr Infect Dis J 2019;38:710-5.

12 Lineberry N, Berlin JA, Mansi B, et al. Recommendations to improve adverse event reporting in clinical trial publications: a joint pharmaceutical industry/journal editor perspective. BMJ 2016;355.

13 Haidich A-B, Birtsou C, Dardavessis T, et al. The quality of safety reporting in trials is still suboptimal: survey of major general medical journals. J Clin Epidemiol 2011;64:124-35.

14 US department of health and human services, National Institutes of health, National Institute of allergy and infectious diseases, division of AIDS. division of AIDS (DAIDS) table for grading the severity of adult and pediatric adverse events, corrected version 2.1, 2017. Available: https:// https://rsc.niaid.nih.gov/sites/default/files/daidsgra dingcorrectedv21.pdf [Accessed 14 Aug 2019].

15 Flahault A, Cadilhac M, Thomas G. Sample size calculation should be performed for design accuracy in diagnostic test studies. J Clin Epidemiol 2005;58:859-62.

16 Hanley JA, Lippman-Hand A. If nothing goes wrong, is everything all right? Interpreting zero numerators. JAMA 1983;249:1743-5.

17 Saint-Raymond A, Hill S, Martines J, et al. CONSORT 2010. Lancet 2010;376:229-30.

18 Fitchett EJA, Seale AC, Vergnano S, et al. Strengthening the reporting of observational studies in epidemiology for newborn infection (STROBE-NI): an extension of the STROBE statement for neonatal infection research. Lancet Infect Dis 2016;16:e202-13.

19 Basmaci R, Vazouras K, Bielicki J, et al. Urinary tract infection antibiotic trial study design: a systematic review. Pediatrics 2017;140:e20172209.

20 European Medicines Agency. Report on the expert meeting on neonatal and paediatric sepsis (EMA/477725/2010). Available: http:// www.ema.europa.eu/docs/en_GB/document_library/Report/2010/12/ WC500100199.pdf [Accessed 14 Aug 2019].

21 Goldstein B, Giroir B, Randolph A, et al. International pediatric sepsis consensus conference: definitions for sepsis and organ dysfunction in pediatrics. Pediatr Crit Care Med 2005;6:2-8. 